\title{
Principles of Quantum Mechanics and Laws of Wave Optics from One Mathematical Formula
}

\author{
Do Tan Si $\mathbb{1}$ \\ Ho Chi Minh City Physical Association, Ho Chi Minh City, Vietnam \\ Email: tansi_do@yahoo.com
}

How to cite this paper: $\mathrm{Si}$, D.T. (2019) Principles of Quantum Mechanics and Laws of Wave Optics from One Mathematical Formula. Applied Mathematics, 10, 892-906.

https://doi.org/10.4236/am.2019.1011064

Received: October 11, 2019

Accepted: November 8, 2019

Published: November 11, 2019

Copyright $\odot 2019$ by author(s) and Scientific Research Publishing Inc. This work is licensed under the Creative Commons Attribution International License (CC BY 4.0).

http://creativecommons.org/licenses/by/4.0/

\begin{abstract}
Finding that in the formula of expansion of a function $f(\vec{r})$ into a series of wave-like functions $\exp (i \overrightarrow{\boldsymbol{k}} \overrightarrow{\boldsymbol{r}})$ the coefficients are its Fourier transforms, if existed, we deduce mathematically all the principles and hypothesis that illustrated physicists utilized to build quantum mechanics a century ago, beginning with the duality particle-wave principle of Planck and including the Schrödinger equations. By the way, we find a simple Fourier transform relation between Dirac momentum and position bras and a useful permutation relation between operators in phase and Hilbert spaces. Moreover, from the found particle-wave duality formula we prove and obtain again essentially by mathematical analysis all the laws of wave optics concerning reflections, refractions, polarizations, diffractions by one or many identical 3D objects with various forms and dimensions.
\end{abstract}

\section{Keywords}

Fourier Transform in Quantum Mechanics, Permutation Relations between Operators, Laws of Wave Optics, Diffractions by Multiform Identical Objects

\section{Introduction}

From the find that a function $f(\overrightarrow{\boldsymbol{r}})$ may be expanded into a series of functions $\mathrm{e}^{i \vec{k} \vec{r}}$ with coefficients equal to $(2 \pi)^{3 / 2}$ multiplies the Fourier transform $\tilde{f}(\overrightarrow{\boldsymbol{k}})$ of $f(\vec{r})$ we arrive to obtain that a particle moving with celerity $\overrightarrow{\boldsymbol{v}}_{0}$, momentum $\overrightarrow{\boldsymbol{p}}_{0}$ creates a wave, confirming the wave-particle duality principle conceived by Planck and Einstein in 1900-1905. Moreover we obtain that $p_{0}$ is inversely proportional to the wavelength of this wave conformed with the hypothesis of de Broglie and that the particle's energy is proportional to the wave's frequency conformed with the proposition of Planck. The coefficient of propor- 
tionality is then identifiable with the Planck's constant $h$.

The Exclusion principle of Pauli may be explained by the assimilation of two particles having the same momentum and the same position with only one having double momentum so that the de Broglie wavelength is divided by two which is a paradox.

From the fact that $\delta\left(\overrightarrow{\boldsymbol{p}}-\overrightarrow{\boldsymbol{p}}_{0}\right)$ represents the momentum-representation of the state $\left|\overrightarrow{\boldsymbol{p}}_{0}\right\rangle$ and $(2 \pi)^{-\frac{3}{2}} \mathrm{e}^{i \hbar^{-1} \overrightarrow{\boldsymbol{p}}_{0} \overrightarrow{\boldsymbol{r}}}$ its position-representation we obtain the relation $\langle\overrightarrow{\boldsymbol{k}}|=F T\langle\overrightarrow{\boldsymbol{r}}|$ where $\overrightarrow{\boldsymbol{p}}=\hbar \overrightarrow{\boldsymbol{k}}$. These relations lead to the canonical commutation relations $\left[\hat{\boldsymbol{r}}_{j}, \hat{\boldsymbol{p}}_{l}\right]=i \hbar \delta_{j l} \hat{I}, E=i \hbar \partial_{t}$ of Born which in turn lead to the well known Schrödinger equations. Utilizing the relation $\langle\vec{k}|=F T\langle\overrightarrow{\boldsymbol{r}}|$ we see also that the Heisenberg's incertitude relation $\Delta x \Delta p>\hbar / 2$ is a matter of Fourier transform relation between the rectangular function $a^{-1}(H(k+a)-H(k-a))$ and the function $\sin (a x) /(a x), H(x)$ being the Heaviside function.

Consider an atom having a discrete spectrum of states each having a value of energy $E_{j}$. It is represented by $\langle E \mid \alpha\rangle=\sum^{N} \delta\left(E-E_{j}\right)$. By searching the maximum values of $|\langle t \mid \alpha\rangle|^{2}$ we see that from time to time there have emission/absorption of a wave having frequency $v_{j k}=h^{-1}\left(E_{k}-E_{j}\right)$ conformed with the theory of Bohr. Besides we obtain permutation relations between functions of creation and annihilation operators in second quantization.

By the same formula giving quantum mechanics' principles we realize that the product of a wave $\mathrm{e}^{i \vec{k}_{0} \vec{r}}$ and an object described by a function $f(\vec{r})$ is a sum over $\mathrm{e}^{i \overrightarrow{\boldsymbol{k}} \vec{r}}$ with coefficients equal to $(2 \pi)^{3 / 2} \tilde{f}\left(\overrightarrow{\boldsymbol{k}}-\overrightarrow{\boldsymbol{k}}_{0}\right)$. This opens a simple way to calculate the amplitude of diffraction of a wave by a $3 \mathrm{D}$ object such as a semi-space which leads to the Descartes, Snell's laws, Fresnel equations, then by a set of identical objects having different geometric forms such as plane which leads to the Braag's formula, pyramid, sphere, etc.

Details of the finds are explained successively in the following paragraphs.

\section{Obtaining Principles and Hypothesis of Quantum Mechanics}

\subsection{The Wave-Particle Duality Principle}

Let us expand a function $f(\vec{r})$ having Fourier transform on a basis of exponential functions

$$
f(\overrightarrow{\boldsymbol{r}})=\sum_{\vec{k}} c(\overrightarrow{\boldsymbol{k}}) \mathrm{e}^{i \overrightarrow{\boldsymbol{k}} \overrightarrow{\boldsymbol{r}}}
$$

where $\overrightarrow{\boldsymbol{k}}$ belongs to an infinite set of vectors obeying the condition that the scalar product $\overrightarrow{\boldsymbol{k}} \overrightarrow{\boldsymbol{r}}$ is dimensionless for the following relation to hold

$$
\mathrm{e}^{i \vec{k} \vec{r}}=1 \cdot \mathrm{e}^{i \vec{k} \vec{r}}=\mathrm{e}^{i(\vec{k} \vec{r}+2 \pi)}
$$

Under such condition we may write 


$$
\begin{aligned}
\int_{R^{3}} \mathrm{e}^{-i \overrightarrow{\boldsymbol{k}}_{0} \vec{r}} f(\overrightarrow{\boldsymbol{r}}) \mathrm{d} \overrightarrow{\boldsymbol{r}} & =\sum_{\vec{k}} c(\overrightarrow{\boldsymbol{k}}) \int_{R^{3}} \mathrm{e}^{-i \overrightarrow{\boldsymbol{k}}_{0} \vec{r}} \mathrm{e}^{i \overrightarrow{\boldsymbol{k}} \vec{r}} \mathrm{~d} \overrightarrow{\boldsymbol{r}} \\
& =(2 \pi)^{3} \sum_{\vec{k}} c(\overrightarrow{\boldsymbol{k}}) \delta\left(\overrightarrow{\boldsymbol{k}}-\overrightarrow{\boldsymbol{k}}_{0}\right) \\
& =(2 \pi)^{3} c\left(\overrightarrow{\boldsymbol{k}}_{0}\right)
\end{aligned}
$$

so that we may state the theorem:

"Any function $f(\overrightarrow{\boldsymbol{r}})$ having Fourier transform may be written under the form

$$
f(\overrightarrow{\boldsymbol{r}})=(2 \pi)^{3 / 2} \sum_{\overrightarrow{\boldsymbol{k}}} \tilde{f}(\overrightarrow{\boldsymbol{k}}) \mathrm{e}^{i \overrightarrow{\boldsymbol{k}} \vec{r}}
$$

where $\overrightarrow{\boldsymbol{k}} \overrightarrow{\boldsymbol{r}}$ is dimensionless and $\tilde{f}(\overrightarrow{\boldsymbol{k}})$ is the Fourier transform of $f(\overrightarrow{\boldsymbol{r}})$

$$
\tilde{f}(\overrightarrow{\boldsymbol{k}})=\operatorname{FTf}(\overline{\boldsymbol{r}})=(2 \pi)^{-3 / 2} \int_{R^{3}} \mathrm{e}^{-i \overrightarrow{\boldsymbol{k}} \overrightarrow{\boldsymbol{r}}} f(\overrightarrow{\boldsymbol{r}}) \mathrm{d} \overrightarrow{\boldsymbol{r}}
$$

Now from the well known formulas

$$
\begin{gathered}
f(x+a)=\mathrm{e}^{a \partial_{x}} f(x) \\
F T D_{x} f(x)=F T f^{\prime}(x)=i k F T f(x)
\end{gathered}
$$

we get

$$
F T \delta(x-a)=F T \mathrm{e}^{-a D_{x}} \delta(x)=\mathrm{e}^{-i a k} F T \delta(x)=\mathrm{e}^{-i a k}(2 \pi)^{-1 / 2}
$$

so that by (2.1.4)

$$
\delta\left(\overrightarrow{\boldsymbol{r}}-\overrightarrow{\boldsymbol{r}}_{0}\right)=\sum_{\vec{k}} \mathrm{e}^{-i \overrightarrow{\boldsymbol{k}_{0}} \overrightarrow{\boldsymbol{r}}} \mathrm{e}^{i \overrightarrow{\boldsymbol{k}} \vec{r}}=\sum_{\vec{k}} \mathrm{e}^{i \vec{k}\left(\vec{r}-\vec{r}_{0}\right)}
$$

Consider a particle situated at the position $\overrightarrow{\boldsymbol{r}}_{0}$ and having a mass $m$ and a constant celerity $\overrightarrow{\boldsymbol{v}}_{0}$. Defining

$$
\overrightarrow{\boldsymbol{k}}_{0}=\frac{2 \pi}{\lambda_{0}} \frac{\overrightarrow{\boldsymbol{v}}_{0}}{v_{0}}=\frac{2 \pi}{\lambda_{0}} \overrightarrow{\boldsymbol{n}}
$$

where $\lambda_{0}$ has the dimension of a length as it must be for $\overrightarrow{\boldsymbol{k}}_{0} \overrightarrow{\boldsymbol{r}}$ to be dimensionless we see from (2.1.9) that the formula

$$
\delta\left(\overrightarrow{\boldsymbol{k}}-\overrightarrow{\boldsymbol{k}}_{0}\right) \delta\left(\overrightarrow{\boldsymbol{r}}-\overrightarrow{\boldsymbol{r}}_{0}\right)=\mathrm{e}^{i \overrightarrow{\boldsymbol{k}}_{0}\left(\overrightarrow{\boldsymbol{r}}-\overrightarrow{\boldsymbol{r}}_{0}\right)}=\exp i\left(\frac{2 \pi}{\lambda_{0}}\left(\overrightarrow{\boldsymbol{r}}-\overrightarrow{\boldsymbol{r}}_{0}\right) \overrightarrow{\boldsymbol{n}}\right)
$$

represents at the same time this particle and a wave. Thank to the property $\mathrm{e}^{ \pm i 2 \pi}=1$ this wave has a wavelength $\lambda_{0}$ and consequently a period

$$
T_{0}=\lambda_{0} / v_{0}
$$

The wave function of this particle is then within a multiplicative constant

$$
\Psi_{0}(\vec{r}, t)=A \exp i\left(\overrightarrow{\boldsymbol{k}}_{0}\left(\overrightarrow{\boldsymbol{r}}-\overrightarrow{\boldsymbol{r}}_{0}\right)-\frac{2 \pi}{T_{0}} t\right)
$$

This is the insight of the principle of wave-particle duality conceived by Planck in 1900 [1] and Einstein in 1905 [2]. It constitutes the first quantization of quantum mechanics. 


\subsection{The de Broglie Particle-Wave Hypothesis and the Planck-Einstein Relation}

As

$$
\overrightarrow{\boldsymbol{k}} / / \overrightarrow{\boldsymbol{v}} / / \overrightarrow{\boldsymbol{p}}=m \overrightarrow{\boldsymbol{v}}
$$

we may define a universal constant $\theta$ having dimension $M L^{2} T^{-1}$ then link $\vec{p}$ with $\overrightarrow{\boldsymbol{k}}$ by the relation

$$
\overrightarrow{\boldsymbol{p}}=\theta \overrightarrow{\boldsymbol{k}}=\theta \frac{2 \pi}{\lambda} \frac{\overrightarrow{\boldsymbol{v}}}{v}=\theta \frac{2 \pi}{\lambda} \overrightarrow{\boldsymbol{n}}
$$

in order to get the form of the relation between momentum and associated wavelength

$$
p=\theta k=\theta \frac{2 \pi}{\lambda}
$$

in accordance with the hypothesis proposed in 1923 by de Broglie [3].

The wave function of the considered particle may then be put under the form

$$
\Psi_{0}(\overrightarrow{\boldsymbol{r}}, t)=A \exp i \theta^{-1}\left(\overrightarrow{\boldsymbol{p}}_{0}\left(\overrightarrow{\boldsymbol{r}}-\overrightarrow{\boldsymbol{r}}_{0}\right)-\frac{2 \pi \theta}{T_{0}} t\right)
$$

By dimensional consideration we see that the quantity $\frac{2 \pi \theta}{T_{0}}$ is an energy that we baptize $E_{0}$ and propose to assimilate it with the energy of the quoted particle

$$
E_{0}=\frac{2 \pi \theta}{T_{0}}
$$

By comparison with the formulae of Planck-Einstein [1] [2] and de Broglie [3]

$$
E=\frac{h}{T}, \quad p=\frac{h}{\lambda}
$$

we get the identifications

$$
\begin{gathered}
\theta=\frac{h}{2 \pi}=\hbar \\
\overrightarrow{\boldsymbol{p}}=\hbar \overrightarrow{\boldsymbol{k}}
\end{gathered}
$$

and see that $\overrightarrow{\boldsymbol{k}}$ is the commonly called wave-vector of a wave.

From now all we say that $\overrightarrow{\boldsymbol{k}}$ and $\overrightarrow{\boldsymbol{r}}$ are Fourier transform reciprocal as so as $\frac{2 \pi}{T}=\hbar^{-1} E$ and the time $t$. The Planck constant $h$ was measured by Millikan [4] in 1916. The best current value for $h$ is $6.62607004 \times 10^{-34} \mathrm{~m}^{2} \cdot \mathrm{kg} / \mathrm{sec}$ and is officially utilized from the date 20-05-2019 on to define the value of the kilogram.

\subsection{The Pauli Exclusion Principle}

A consequence of the relation (2.2.4) and the de Broglie hypothesis (2.2.6) we see that if two particles have the same value of momentum $\vec{p}$ and the same posi- 
tion they may be assimilated to one particle with momentum $2 \overrightarrow{\boldsymbol{p}}$ so that the dual wave must have its wavelength divided by 2 . This leads to a paradox and confirms the Exclusion principle of Pauli [5]. For photons with momentum $p=h v / c$ too small, two times of it is quasi equal to it so that there is no paradox, i.e. many photons may occupy one position.

\subsection{Obtaining the Fourier Transform Relation between Bras $\langle\vec{k}|$ and $\langle\vec{r}|$}

In a Hilbert space of Dirac kets and bras let according to (2.1.13)

$$
\left\langle\overrightarrow{\boldsymbol{r}} \mid \overrightarrow{\boldsymbol{k}}_{0}\right\rangle=(2 \pi)^{-3 / 2} \exp \left(i \overrightarrow{\boldsymbol{k}}_{0} \overrightarrow{\boldsymbol{r}}\right)
$$

be the position-representation of a state having a definite wave-vector $\overrightarrow{\boldsymbol{k}}_{0}$. From the formula

$$
F T \mathrm{e}^{i \overrightarrow{\boldsymbol{k}}_{0} \vec{r}}=(2 \pi)^{-3 / 2} \int_{R^{3}} \mathrm{e}^{-i\left(\overrightarrow{\boldsymbol{k}}-\overrightarrow{\boldsymbol{k}}_{0}\right) \overrightarrow{\boldsymbol{r}}} \mathrm{d} \overrightarrow{\boldsymbol{r}}=(2 \pi)^{3 / 2} \delta\left(\overrightarrow{\boldsymbol{k}}-\overrightarrow{\boldsymbol{k}}_{0}\right)
$$

and (2.4.1) we have

$$
F T\left\langle\overrightarrow{\boldsymbol{r}} \mid \overrightarrow{\boldsymbol{k}}_{0}\right\rangle=F T(2 \pi)^{-3 / 2} \mathrm{e}^{i \overrightarrow{\boldsymbol{k}}_{0} \vec{r}}=\delta\left(\overrightarrow{\boldsymbol{k}}-\overrightarrow{\boldsymbol{k}}_{0}\right)=\left\langle\overrightarrow{\boldsymbol{k}} \mid \overrightarrow{\boldsymbol{k}}_{0}\right\rangle
$$

so that, because $\overrightarrow{\boldsymbol{k}}_{0}$ is arbitrary, we get the interesting relation

$$
\langle\overrightarrow{\boldsymbol{k}}|=F T\langle\overrightarrow{\boldsymbol{r}}|
$$

which gives precision to the latent idea in many researchers that there exists somehow a Fourier relation between momentum and position:

"In quantum mechanics the wave-vector bra $\langle\overrightarrow{\boldsymbol{k}}|$ is the Fourier transform of the position bra $\langle\overrightarrow{\boldsymbol{r}}|$ ".

From (2.4.4) we get the relation between momentum-representation and position-representation of a state

$$
\langle\overrightarrow{\boldsymbol{k}} \mid \Psi\rangle=F T\langle\overrightarrow{\boldsymbol{r}} \mid \Psi\rangle
$$

\subsection{The Canonical Commutation Postulated by Born}

In the Hilbert space of states besides $\hat{X}$ and $\hat{P}_{x}$ let us formally define another operator $\hat{D}_{x}$

by the relation

$$
\hat{D}_{x} \hat{X}-\hat{X} \hat{D}_{x} \equiv \hat{I}
$$

where $\hat{I}$ is the identity operator.

Now, in the space of functions let $\breve{X}$ be the operator of multiplication by $x$ and $\breve{D}_{x}$ the derivative operator

$$
\breve{X} f(x)=x f(x) ; \breve{D}_{x} f(x)=f^{\prime}(x)
$$

verifying

$$
\left[\breve{D}_{x}, \breve{X}\right] \equiv \breve{D}_{x} \breve{X}-\breve{X} \breve{D}_{x} \equiv \breve{I}
$$


We must be attentive on the fact that the operators $\breve{X}, \breve{D}_{x}, \breve{P}_{x}, \breve{D}_{p}$ act on functions and $\hat{X}, \hat{D}_{x}, \hat{P}_{x}, \hat{D}_{p_{x}}$ act on bras and kets.

From (2.5.1), (2.5.3) we get

$$
\begin{gathered}
\left\langle x\left|\hat{D}_{x} \hat{X}-\hat{X} \hat{D}_{x}\right| x_{10}\right\rangle=\left(x_{10}-x\right)\left\langle x\left|\hat{D}_{x}\right| x_{0}\right\rangle=\delta\left(x-x_{10}\right) \\
\breve{D}_{x}\left(\breve{X}-x_{0}\right) \delta\left(x-x_{10}\right)=0 \\
\left(\breve{D}_{x} \breve{X}-\breve{X} \breve{D}_{x}\right) \delta\left(x-x_{10}\right)=\left(x_{0}-x\right) \breve{D}_{x} \delta\left(x-x_{10}\right)=\delta\left(x-x_{10}\right)
\end{gathered}
$$

so that

$$
\left\langle x\left|\hat{D}_{x}\right| x_{0}\right\rangle=\breve{D}_{x}\left\langle x \mid x_{0}\right\rangle
$$

Besides we have also

$$
\left\langle x|\hat{X}| x_{0}\right\rangle=x \delta\left(x-x_{0}\right)=\breve{X}\left\langle x \mid x_{0}\right\rangle
$$

so that, as $x_{0}$ is arbitrary,

$$
\langle x| \hat{D}_{x} \equiv \breve{D}_{x}\langle x| ;\langle x| \hat{X} \equiv \breve{X}\langle x|
$$

The above relations associated with (2.4.4) and

$$
F T x f(x)=(2 \pi)^{-1 / 2} \int_{-\infty}^{\infty} i \partial_{k} \mathrm{e}^{-i k x} f(x) \mathrm{d} x=i \partial_{k} F(x)
$$

lead to

$$
\begin{aligned}
\left\langle k|\hat{X}| k_{0}\right\rangle & =F T\left\langle x|\hat{X}| k_{0}\right\rangle=F T x\left\langle x \mid k_{0}\right\rangle \\
& =i \partial_{k} F T\left\langle x \mid k_{0}\right\rangle=i \partial_{k}\left\langle k \mid k_{0}\right\rangle=\left\langle k\left|i \hat{D}_{k}\right| k_{0}\right\rangle
\end{aligned}
$$

i.e.

$$
\hat{X} \equiv i \hat{D}_{k} \equiv i \hbar \hat{D}_{p}
$$

Similarly by repeating the reasoning with $\hat{P}_{x}, \hat{D}_{p_{x}}$ we get

$$
\hat{P}_{x}=-i \hbar \hat{D}_{x}
$$

Extension to 3D space gives

$$
\hat{\boldsymbol{r}} \equiv i \hat{\nabla}_{k} \equiv i \hbar \hat{\nabla}_{p}
$$

and finally the commutation relations

$$
\left[\hat{r}_{j}, \hat{p}_{l}\right]=-i \hbar\left[\hat{r}_{j}, \hat{\nabla}_{l}\right]=i \hbar \delta_{j l} \hat{I}
$$

which have been called quantum conditions and postulated by Born in 1925 [6]. Similarly from the fact that $\frac{2 \pi}{T}=\hbar^{-1} E$ and $t$ are Fourier reciprocal we have

$$
E=i \hbar \partial_{t}
$$

\subsection{The Schrödinger Equations}

From the relations (2.5.6) we may also get an important proposition:

"The eigenvalue equation

$$
A(\hat{X}, \hat{P})|\alpha\rangle=a|\alpha\rangle
$$


of an arbitrary operator $A(\hat{X}, \hat{P})$ leads to the differential equation for the function $\langle x \mid \alpha\rangle$

$$
\langle x|A(\hat{X}, \hat{P})| \alpha\rangle=A\left(\breve{X},-i \hbar \breve{D}_{x}\right)\langle x \mid \alpha\rangle=a\langle x \mid \alpha\rangle
$$

For example, with

$$
A(\hat{X}, \hat{P}) \equiv \frac{1}{2 m} \hat{P}^{2}+V(\hat{X})
$$

we obtain the well known time independent Schrödinger equation [7]

$$
\left(\frac{-\hbar^{2}}{2 m} \breve{D}_{x}^{2}+V(x)\right) \Psi(x)=E \Psi(x)
$$

As $\frac{2 \pi}{T}=\hbar^{-1} E$ and $t$ are Fourier transform reciprocal we get the time dependent Schrödinger equation

$$
\left(-\frac{\hbar^{2}}{2 m} \frac{\partial^{2}}{\partial x^{2}}+V(x, t)\right)\langle x, t \mid \Psi\rangle=i \hbar \partial_{t}\langle x, t \mid \Psi\rangle
$$

\subsection{The Heisenberg Uncertainty Principle}

Let $S(k, \Delta k)$ be the function equal to zero for $|k|>\Delta k / 2$ and to $(\Delta k)^{-1}$ for $|k|<\Delta k / 2$ as illustrated by Figure 1 .

A state $|\alpha\rangle$ where there is incertitude on the wave-number $k$

$$
\left(k_{0}-\Delta k / 2\right) \leq k \leq\left(k_{0}+\Delta k / 2\right)
$$

corresponds to the momentum-representation

$$
\langle k \mid \alpha\rangle=S\left(k-k_{0}, \Delta k\right)=\mathrm{e}^{-k_{0} \partial_{k}} S(k, \Delta k)
$$

Utilizing the Heaviside function we may write

$$
S(k, \Delta k) \equiv \frac{H(k+\Delta k / 2)-H(k-\Delta k / 2)}{\Delta k}
$$

Thank to (2.1.6), (2.1.7) and the property

$$
\begin{aligned}
F T H(k+\Delta k / 2) & =F T \mathrm{e}^{\frac{\Delta k}{2} \partial_{k}} H(k)=\mathrm{e}^{\frac{\Delta k}{2} i x} F T H(k) \\
& =\mathrm{e}^{\frac{\Delta k}{2} i x}(2 \pi)^{-\frac{1}{2}}\left(\frac{1}{i x}+\pi \delta(x)\right)
\end{aligned}
$$

we get by Fourier transform of (2.7.3)

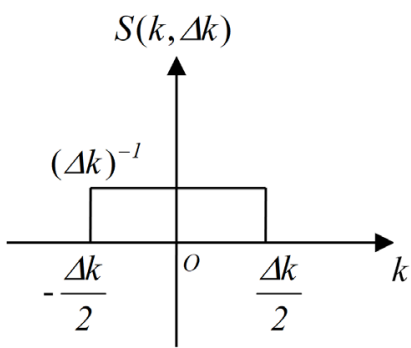

Figure 1. The rectangular function. 


$$
\operatorname{FTS}(k, \Delta k)=(2 \pi)^{-\frac{1}{2}} \frac{\sin (x \Delta k / 2)}{(x \Delta k / 2)}
$$

so that by (2.7.2)

$$
\begin{gathered}
F T F T\langle x \mid \alpha\rangle=F T\langle k \mid \alpha\rangle=F T \mathrm{e}^{-k_{0} \partial_{k}} S(k, \Delta k)=(2 \pi)^{-\frac{1}{2}} \mathrm{e}^{-i k_{0} x} \frac{\sin (x \Delta k / 2)}{(x \Delta k / 2)}(2.7 .6) \\
\langle x \mid \alpha\rangle=F T F T\langle-x \mid \alpha\rangle=(2 \pi)^{-1 / 2} \mathrm{e}^{i k_{0} x} \frac{\sin (x \Delta k / 2)}{(x \Delta k / 2)}
\end{gathered}
$$

The graph of $\langle x \mid \alpha\rangle$ has the form (Figure 2).

The function $\langle x \mid \alpha\rangle$ has maximum value for $x=0$, vanishes for $x \Delta k / 2= \pm \pi$. It and its squared are equal nearly to half of their maxima for $\frac{x \Delta k}{2} \approx \frac{\pi}{2}$ or $x \Delta k \approx \pi$.

We may then write that

$$
\Delta x \Delta p=\hbar \Delta x \Delta k \cong \hbar 2 \pi=h
$$

Because $h>\hbar$ the relation (2.7.6) is conformed with the uncertainty principle announced by Heisenberg [8] and proven somehow by Kennard [9] in 1927.

$$
\Delta x \Delta p \geq \frac{\hbar}{2}
$$

Similarly because the couple $\left(\hbar^{-1} E, t\right)$ are reciprocal so as $\left(\hbar^{-1} p, x\right)$ we get

$$
\Delta t \Delta E \geq \frac{\hbar}{2}
$$

\subsection{Emission of Photons from Atoms Following Bohr}

Consider a state $|\alpha\rangle$ which has many stable values for its energy and suppose that $|\alpha\rangle$ is the sum of individual states each of them having only one value of energy or one frequency

$$
\langle E \mid \alpha\rangle=\sum_{j=1}^{N} \delta\left(E-E_{j}\right)
$$

By Fourier transform we get

$$
\langle t \mid \alpha\rangle=(2 \pi)^{-\frac{1}{2}} \sum_{j=1}^{N} \mathrm{e}^{-i E_{j} t / \hbar}
$$

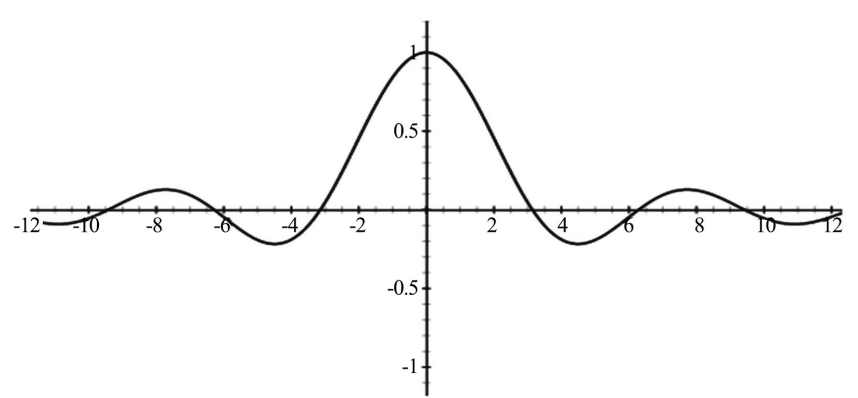

Figure 2. Graph of $\sin (x) / x$. 
so that

$$
|\langle t \mid \alpha\rangle|^{2}=(2 \pi)^{-1}\left(N+\sum_{j=1}^{N} \sum_{k<j} 2 \cos \left(E_{k}-E_{j}\right) t / \hbar\right)
$$

By (2.8.3) we see that the probability for observing $|\alpha\rangle$ at the instant $t$ is maximal for

$$
t_{n}=n \frac{h}{E_{k}-E_{j}}=\frac{n}{v_{k}-v_{j}}, \forall j<k=1,2, \cdots, N ; n=0,1,2, \cdots
$$

In other word we see that from time to time there may have emission/absorption of waves with frequencies

$$
v_{j k}=h^{-1}\left(E_{k}-E_{j}\right)
$$

This result accords with the theory on the constitution of atoms and molecules of Bohr [10] in 1913.

\subsection{Obtaining Permutation Relations between Functions of Creation and Annihilation Operators}

Let $A, B$ be two operators obeying the condition

$$
A B \equiv B A+I
$$

We have

$$
A^{m} B \equiv A A \cdots A B \equiv B A^{m}+m A^{m-1}
$$

because at each time we change $A B$ into $B A$ we must add $A^{m-1}$.

So, let $f(t)$ be an entire function and $f^{\prime}(t)$ its derivative function we clearly have

$$
f(A) B \equiv B f(A)+f^{\prime}(A)
$$

Now from (2.9.3)

$$
f(A) B^{2} \equiv B f(A) B+f^{\prime}(A) B \equiv B^{2} f(A)+2 B f^{\prime}(A)+f^{\prime \prime}(A)
$$

so that by recursion we get

$$
f(A) B^{m} \equiv \sum_{k=0}^{m}\left(\begin{array}{l}
m \\
k
\end{array}\right) B^{m-k} f^{(k)}(A)
$$

From (2.9.5) we can't sum over $B^{m}$ because of the mixed coefficient $\left(\begin{array}{c}m \\ k\end{array}\right)$ under the summation. After thinking we replace (2.9.5) with the following formula

$$
f(A) B^{m} \equiv \sum_{k=0}^{m} \frac{1}{k !}\left(B^{m}\right)^{(k)} f^{(k)}(A)
$$

so that if $g(B)$ is an entire function we get the fundamental identity between operators obeying the sole condition $A B-B A \equiv I$

$$
f(A) g(B) \equiv \sum_{k=0}^{\infty} \frac{1}{k !} g^{(k)}(B) f^{(k)}(A)
$$

and its dual 


$$
f(B) g(A) \equiv \sum_{k=0}^{\infty}(-1)^{k} \frac{1}{k !} g^{(k)}(A) f^{(k)}(B)
$$

For examples we have successively

$$
\begin{gathered}
\mathrm{e}^{\alpha D_{x}} X \equiv X \mathrm{e}^{\alpha D_{x}}+\alpha \mathrm{e}^{\alpha D_{x}} \equiv(X+\alpha I) \mathrm{e}^{\alpha D_{x}} \\
\mathrm{e}^{\alpha D_{x}} f(X) \mathrm{e}^{-\alpha D_{x}} \equiv f(X+\alpha I) \\
\alpha D_{x} \mathrm{e}^{\frac{\beta}{2 \alpha} X^{2}} \equiv \mathrm{e}^{\frac{\beta}{2 \alpha} X^{2}}\left(\alpha D_{x}+\beta X\right) \\
\mathrm{e}^{\left(\alpha D_{x}+\beta X\right)} \equiv \mathrm{e}^{-\frac{\beta}{2 \alpha} X^{2}} \mathrm{e}^{\alpha D_{x}} \mathrm{e}^{\frac{\beta}{2 \alpha} X^{2}} \equiv \mathrm{e}^{-\frac{\beta}{2 \alpha} X^{2}} \mathrm{e}^{\frac{\beta}{2 \alpha}(X+\alpha I)^{2}} \mathrm{e}^{\alpha D_{x}} \equiv \mathrm{e}^{\frac{1}{2} \alpha \beta} \mathrm{e}^{\beta X} \mathrm{e}^{\alpha D_{x}}
\end{gathered}
$$

Defining the creation and the annihilation operators by

$$
a^{ \pm} \equiv \frac{1}{\sqrt{2}}\left(D_{x} \mp X\right) \Rightarrow a^{+} a^{-}-a^{-} a^{+} \equiv D_{x} X-X D_{x} \equiv I
$$

we get from (2.9.8), (2.9.9), (2.9.10),

$$
\begin{array}{r}
f\left(a^{-}\right) g\left(a^{+}\right) \equiv \sum_{k=0}^{\infty}(-1)^{k} \frac{1}{k !} g^{(k)}\left(a^{+}\right) f^{(k)}\left(a^{-}\right) \\
\mathrm{e}^{\lambda a^{ \pm}} f(x)=\mathrm{e}^{\mp \frac{1}{4} \lambda^{2}} \mathrm{e}^{\mp \frac{\lambda}{\sqrt{2}} x} f\left(x+\frac{\lambda}{\sqrt{2}}\right), \quad x \in C
\end{array}
$$

Closing this paragraph we propose from (2.9.6) the new version of the Newton's binomial formula

$$
(x+y)^{r}=\sum_{k=0}^{\infty}\left(\begin{array}{l}
r \\
k
\end{array}\right) x^{r-k} y^{k}=\sum_{k=0}^{\infty} \frac{1}{k !} y^{k} D_{x}^{k} x^{r}=\mathrm{e}^{y D_{x}} x^{r}
$$

\section{Obtaining Laws of Wave Optics}

\subsection{Diffraction by a 3D Object Centered at the Origin of Axis System}

Consider an object occupied a limited domain $D$ in space and represented by the object function which may be discontinuous

$$
f_{D}(\overrightarrow{\boldsymbol{r}})=1 \text { for } \overrightarrow{\boldsymbol{r}} \in D \& f_{D}(\overrightarrow{\boldsymbol{r}})=0 \text { for } \overrightarrow{\boldsymbol{r}} \notin D
$$

From the formula (2.1.4) we see that the coexistence of a wave and this object may be represented by

$$
f_{D}(\overrightarrow{\boldsymbol{r}}) \mathrm{e}^{i \overrightarrow{\boldsymbol{k}}_{0} \vec{r}}=(2 \pi)^{3 / 2} \sum_{\vec{k}}\left(F T \mathrm{e}^{i \overrightarrow{\boldsymbol{k}}_{0} \vec{r}} f_{D}(\overrightarrow{\boldsymbol{r}})\right) \mathrm{e}^{i \vec{k} \vec{r}}=(2 \pi)^{3 / 2} \sum_{\vec{k}} \tilde{f}_{D}\left(\overrightarrow{\boldsymbol{k}}-\overrightarrow{\boldsymbol{k}}_{0}\right) \mathrm{e}^{i \overrightarrow{\boldsymbol{k}} \vec{r}}
$$

Equation (3.1.2) gives rise to the main theorem in wave optics

"The amplitude of diffraction of a wave $\overrightarrow{\boldsymbol{k}}_{0}$ into a wave $\overrightarrow{\boldsymbol{k}}$ by the form of an object is equal to $(2 \pi)^{3 / 2}$ multiplies the Fourier transform of the object function calculated for the deviation of the wave-vector $\left(\overrightarrow{\boldsymbol{k}}-\overrightarrow{\boldsymbol{k}}_{0}\right)$ ".

\subsection{Diffraction by Systems of Identical Objects Centered at the Positions $\vec{r}_{j}$}

Consider a set of objects centered at the points $\overrightarrow{\boldsymbol{r}}_{j}$. Utilizing (2.1.6), (2.1.7) we 
have

$$
\begin{gathered}
f_{D}\left(\overrightarrow{\boldsymbol{r}}-\overrightarrow{\boldsymbol{r}}_{j}\right)=\mathrm{e}^{-\overrightarrow{\boldsymbol{r}}_{j} \nabla_{r}} f_{D}(\overrightarrow{\boldsymbol{r}}) \\
F T f_{D}\left(\overrightarrow{\boldsymbol{r}}-\overrightarrow{\boldsymbol{r}}_{j}\right)=F T \mathrm{e}^{-\overrightarrow{\boldsymbol{r}}_{j} \nabla_{\vec{r}}} f_{D}(\overrightarrow{\boldsymbol{r}})=\mathrm{e}^{-i \overrightarrow{\overrightarrow{\boldsymbol{k}}_{j}} \tilde{f}_{D}} \tilde{f}_{D}(\overrightarrow{\boldsymbol{k}})
\end{gathered}
$$

and get a useful formula giving the amplitude of diffraction in some direction $\overrightarrow{\boldsymbol{k}}$ of a plane wave $\overrightarrow{\boldsymbol{k}}_{0}$ by a set of identical objects

$$
(2 \pi)^{3 / 2} \sum_{j} F T f_{D}\left(\overrightarrow{\boldsymbol{r}}-\overrightarrow{\boldsymbol{r}}_{j}\right)=(2 \pi)^{3 / 2} \tilde{f}_{D}(\Delta \overrightarrow{\boldsymbol{k}}) \sum_{j} \exp \left(-i \Delta \overrightarrow{\boldsymbol{k}} \overrightarrow{\boldsymbol{r}}_{j}\right)
$$

\subsection{Applications}

\subsubsection{Diffraction of $\vec{k}_{0}$ by a Semi Space}

The semi space under the plane $O x y$ is described by the object function

$$
f_{O x y}(\overrightarrow{\boldsymbol{r}})=u(x) u(y) H(-z), u(x)=1
$$

From the theorem (2.1.4) we see that

$$
f_{O x y}(\overrightarrow{\boldsymbol{r}}) \mathrm{e}^{i \overrightarrow{\boldsymbol{k}}_{0} \vec{r}}=(2 \pi) \sum_{\vec{k}} \delta\left((\Delta \overrightarrow{\boldsymbol{k}})_{x}\right) \delta\left((\Delta \overrightarrow{\boldsymbol{k}})_{y}\right) \tilde{H}\left(-(\Delta \overrightarrow{\boldsymbol{k}})_{z}\right) \mathrm{e}^{i \overrightarrow{\boldsymbol{k}} \vec{r}}
$$

so that there are diffracted waves only for

$$
\begin{gathered}
(\Delta \overrightarrow{\boldsymbol{k}})_{x}=(\Delta \overrightarrow{\boldsymbol{k}})_{y}=0 \\
\overrightarrow{\boldsymbol{k}}_{x}^{\prime \prime}-\overrightarrow{\boldsymbol{k}}_{0 x}=\overrightarrow{\boldsymbol{k}}_{y}^{\prime \prime}-\overrightarrow{\boldsymbol{k}}_{0 y}=\overrightarrow{\boldsymbol{k}}_{x}^{\prime}-\overrightarrow{\boldsymbol{k}}_{0 x}=\overrightarrow{\boldsymbol{k}}_{y}^{\prime}-\overrightarrow{\boldsymbol{k}}_{0 y}=0
\end{gathered}
$$

Equations (3.3.3) gives the Descartes law of reflection [11] which implies that $\overrightarrow{\boldsymbol{k}}_{0}$ and $\overrightarrow{\boldsymbol{k}}^{\prime \prime}$ must be symmetric as shown Figure 3. Moreover if the diffracted wave $\overrightarrow{\boldsymbol{k}}^{\prime}$ is situated in a medium where the refractive index is $n$ so that $k^{\prime}=n k_{0} \quad$ we get the Snell's law for refraction [11]

$$
k_{x}^{\prime}-k_{0 x}=k_{0}(n \sin r-\sin i)=0
$$

\subsubsection{Obtaining the Fresnel Formulae}

Now, let $a, a^{\prime}, a^{\prime \prime}$ denoted the amplitudes of the incident, the refracted and the reflected waves; $n_{1}, n_{2}$ the upper and lower semi-space refraction indices.

The amplitudes $a^{\prime}, a^{\prime \prime}$ are proportional to $a$ and respectively to

$$
\tilde{f}_{D}\left(0,0, \Delta \overrightarrow{\boldsymbol{k}}_{z}^{\prime}\right), \quad \tilde{f}_{D}\left(0,0, \Delta \overrightarrow{\boldsymbol{k}}_{z}^{\prime \prime}\right)
$$

Remarking that the Fourier transform of a Heaviside function $H(z)$ is

$$
\tilde{H}\left(k_{z}\right)=(2 \pi)^{-1 / 2}\left(\frac{1}{i k_{z}}+\pi \delta\left(k_{z}\right)\right)
$$

we get

$$
\begin{aligned}
& a^{\prime}=\frac{v a}{\left(\vec{k}^{\prime}-\vec{k}_{0}\right)_{z}}=\frac{v a}{k^{\prime} \cos r-k_{0} \cos i}=\frac{v a \sin r}{k_{0} \sin (i-r)} \\
& a^{\prime \prime}=\mu a \frac{1}{\left(\vec{k}^{\prime \prime}-\vec{k}_{0}\right)_{z}}=-\frac{\mu a}{2 k_{0} \cos i}
\end{aligned}
$$




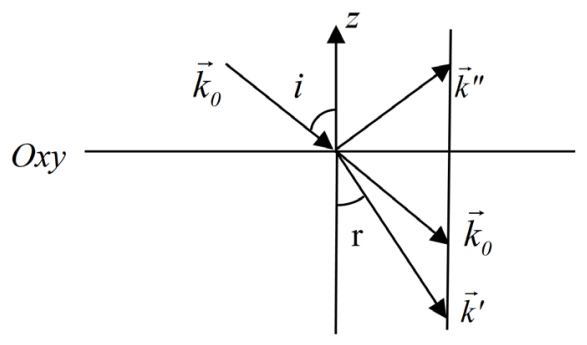

Figure 3. Diffraction by the half space under the plane $O x y$.

In order to calculate the coefficients $\mu, v$ we will make use of the law of conservation of energies. The incoming density of energy at the interface $O x y$ is proportional to $a^{2}$, to the inclination $\left|\cos i_{1}\right|$ and the duration of time an incoming photon is in the vicinity of it, i.e. to $v_{1}^{-1}$ or $n_{1}$. Similarly for the density of outgoing energies so that

$$
n_{1} a^{2} \cos i=n_{2} a^{\prime 2} \cos r+n_{1} a^{\prime \prime 2} \cos i
$$

The above equations and the formula

$$
4 \cos i \sin i \cos r \sin r=\sin ^{2}(i+r)-\sin ^{2}(i-r)
$$

lead by (3.2.3) to the following

$$
4 k_{0}^{2} n_{1}^{2} \cos ^{2} i \sin ^{2}(i-r)=\mu^{2} \sin ^{2}(i-r)+v^{2}\left(\sin ^{2}(i+r)-\sin ^{2}(i-r)\right)
$$

- Taken $v=0$ we get $\mu=2 k_{0} n_{1} \cos i$ and there is total reflection.

- Taken $\mu=v=2 n_{1} k_{0} \cos i \sin (i-r) / \sin (i+r)$ we get the Fresnel formulae [11]

$$
\begin{aligned}
& \frac{a^{\prime}}{a}=\frac{2 n_{1} \cos i}{n_{1} \cos i+n_{2} \cos r}=\frac{2 \cos i \sin r}{\sin (i+r)} \\
& \frac{a^{\prime \prime}}{a}=\frac{n_{1} \cos i-n_{2} \cos r}{n_{1} \cos i+n_{2} \cos r}=-\frac{\sin (i-r)}{\sin (i+r)}
\end{aligned}
$$

- Taken $\mu=-v \cos (i+r)$ we get the second Fresnel formulae [11]

$$
\begin{aligned}
\frac{a^{\prime}}{a} & =\frac{2 n_{1} \cos i}{n_{1} \cos i+n_{2} \cos r}=\frac{2 \cos i \sin r}{\sin (i+r) \cos (i-r)} \\
\frac{a^{\prime \prime}}{a} & =\frac{\tan (i-r)}{\tan (i+r)}
\end{aligned}
$$

From (3.3.12) we find again the Brewster's condition for total polarization $(i+r)=\frac{\pi}{2}, \quad a^{\prime \prime}=0 \quad[11]$

\subsubsection{Diffraction by a Sphere}

The equation of a sphere centered at $\mathrm{O}$ and having radius $R$ as shown in Figure 4 is

$$
S(x, y, z)=H\left(R^{2}-z^{2}\right) H\left(R^{2}-y^{2}-z^{2}\right) H\left(R^{2}-x^{2}-y^{2}-z^{2}\right)
$$

Its Fourier transform is invariant in a rotation around the origin so that 


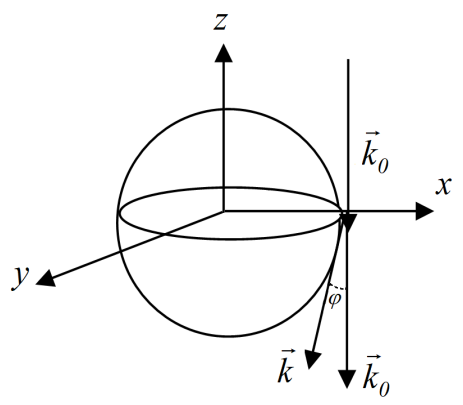

Figure 4. Deflection of waves by a sphere.

$$
\begin{gathered}
\tilde{S}\left(k_{x}, k_{y}, k_{z}\right)=\tilde{S}(0,0, k)=2(2 \pi)^{-3 / 2} \int_{-R}^{R} \mathrm{e}^{-i k z} \mathrm{~d} z \int_{-\sqrt{R^{2}-z^{2}}}^{\sqrt{R^{2}-z^{2}}} \mathrm{~d} y \sqrt{\left(R^{2}-z^{2}\right)-y^{2}} \\
\tilde{S}(\vec{k})=(2 \pi)^{-\frac{1}{2}} \frac{2 R}{k^{2}}\left(\frac{\sin R k}{R k}-\cos R k\right)
\end{gathered}
$$

As conclusion we see that in a diffraction by a sphere the amplitude of diffraction is inversely proportional to $(\Delta k)^{2}$ with $\Delta k=\|\Delta \vec{k}\|$ and there is extinction if

$$
\tan R \Delta k=R \Delta k \Rightarrow R \Delta k=0.02
$$

Let $\varphi$ be the deviation angle in a diffraction as shown Figure 5, we have extinction for $\varphi$ such that

$$
\sin \frac{\varphi}{2}=\frac{\Delta k}{2 k}=\frac{R \Delta k}{2 R k}=\frac{0.02}{2 R k}
$$

For example, for $\lambda=10 \mathrm{~nm}$ and $R=2.5 \mathrm{~nm}$ hemoglobin, there is extinction if

$$
\sin \frac{\varphi}{2}=\frac{0.02}{\pi}=0.0064
$$

\subsubsection{Diffraction of a Plane Wave by Parallel Planes}

From (3.2.3) we obtain for example the amplitudes of diffraction of a plane wave by parallel planes perpendicular to $O z$ at the points $\pm d, \pm 2 d, \cdots, \pm N d$ as shown Figure 6

$$
(2 \pi)^{3 / 2} \sum_{n=1}^{N}\left(\mathrm{e}^{-i n d \Delta \vec{k}_{z}}+\mathrm{e}^{i n d \Delta \vec{k}_{z} n}\right)=2(2 \pi)^{3 / 2} \cos \frac{(N+1) d \Delta \vec{k}_{z}}{2} \frac{\sin \left(N d \Delta \vec{k}_{z} / 2\right)}{\sin \left(d \Delta \vec{k}_{z} / 2\right)}
$$

The maximum amplitudes of diffraction correspond, because $\vec{k}_{0}$ and $\vec{k}$ have opposite projections on $\mathrm{Oz}$ as shown Figure 6, to

$$
\begin{gathered}
\frac{d \Delta \overrightarrow{\boldsymbol{k}}_{z}}{2}=m \pi \Rightarrow d 2 k_{0} \cos \left(O z, \overrightarrow{\boldsymbol{k}}_{0}\right)=2 m \pi \\
2 d \cos \left(O z, \overrightarrow{\boldsymbol{k}}_{0}\right)=2 d \sin \theta=m \frac{2 \pi}{k_{0}}=m \lambda, \quad m \text { integer }
\end{gathered}
$$

The formula (3.3.19) is identical with the Braag's formula [11]. Apart from the above applications of the formula (3.1.2) for studying wave optics we have many other interesting applications in Ref [12]. 


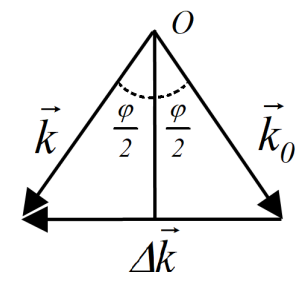

Figure 5. Angle of deflection.

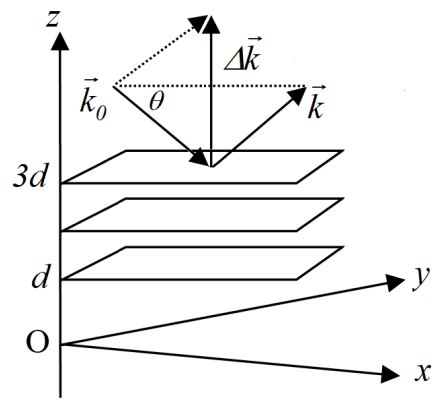

Figure 6. Diffraction by equidistant parallel planes.

\section{Remarks and Conclusions}

Someone has said that "Physics is the studies of Nature, how matter and radiation behave, move and interact thorough space and time. Mathematics, on the other hand, is logical deductive reasoning based on initial assumption. There are many different systems of mathematics that can describe the same physical phenomenon." Accordingly this work which improves and completes a previous work [13] is only one attempt for understanding systematically quasi all the principles and hypothesis of quantum mechanics as so as many aspects of wave optics taught in universities. The main remark is that these quantum principles and laws of optics may be deduced from only one simple formula $f(\overrightarrow{\boldsymbol{r}})=(2 \pi)^{3 / 2} \sum_{\vec{k}} \tilde{f}(\overrightarrow{\boldsymbol{k}}) \mathrm{e}^{i \overrightarrow{\boldsymbol{k}} \vec{r}}$ associated with the property $\mathrm{e}^{ \pm i n 2 \pi}=1$ which leads to quantization. ${ }^{k}$

May this work brings closer students to modern physics!

\section{Acknowledgements}

The author acknowledges Prof. Geneste J.P. for reading and appreciating this work at the World conference on quantum mechanics and nuclear engineering holt in 2019 September at Paris. He thanks warmly the reviewer for giving many judicious remarks and for judging this work as meaningful. He thanks Dr. Feltus Chr. at Luxembourg LIST for laborious writing assistance. He dedicates this work to the Ho Chi Minh-city University of Natural Sciences and the Université libre de Bruxelles where he was formed in the past.

\section{Conflicts of Interest}

The author declares no conflicts of interest regarding the publication of this paper. 


\section{References}

[1] Planck, M. (1901) Ueber das gesetz der energieverteilung im normalspectrum (On the Law of Distribution of Energy in the Normal Spectrum). Annalen der Physik, 309, 553-560. https://doi.org/10.1002/andp.19013090310

[2] Einstein, A. and Infeld, L. (1938) The Evolution of Physics: The Growth of Ideas from Early Concepts to Relativity and Quanta. Cambridge University Press, Cambridge.

[3] de Broglie, L. (1923) Waves and Quanta. Nature, 112, 540. https://doi.org/10.1038/112540a0

[4] Franklina, A. (2013) Millikan's Measurement of Planck's Constant. The European Physical Journal H, 38, 572-594. https://doi.org/10.1140/epjh/e2013-40021-3

[5] Pauli, W. (1925) Über den Zusammenhang des Abschlusses der Elektronengruppen im Atom mit der Komplexstruktur der Spektren. Zeitschrift für Physik, 31, 765-783. https://doi.org/10.1007/BF02980631

[6] Born, M. and Jordan, P. (1925) Zur Quantenmechanik. Zeitschrift für Physik, 34, 858. https://doi.org/10.1007/BF01328531

[7] Griffiths, D.J. (2004) Introduction to Quantum Mechanics. 2nd Edition, Prentice Hall, Upper Saddle River, NJ.

[8] Heisenberg, W. (1927) Über den anschaulichen Inhalt der quantentheoretischen Kinematik und Mechanik. Zeitschrift für Physik, 43, 172-198. https://doi.org/10.1007/BF01397280

[9] Kennard, E.H. (1927) Zur Quantenmechanik einfacher Bewegungstypen. Zeitschrift für Physik, 44, 326-352. https://doi.org/10.1007/BF01391200

[10] Bohr N. (1913) On the Constitution of Atoms and Molecules. The London, Edinburgh, and Dublin Philosophical Magazine and Journal of Science, 26, 1-25. https://doi.org/10.1080/14786441308634955

[11] Bruhat, G. (1965) Optique. Masson \& Cie, Switzerland.

[12] Do Tan, S. (2019) Advances in Optics, Chapter 5. In: The Fourier Transform Relation between $m$ Dirac bras $\langle\vec{k}|=F T\langle\vec{r}|$ and Wave Optics, Reviews Book Series, Volume 4, International Frequency Sensor Association Publishing, Barcelona.

[13] Do Tan, S. (2018) The Fourier Transform and Principles of Quantum Mechanics. Applied Mathematics, 9, 347-354. 